\title{
Refined HLA-DPB1 mismatch with molecular algorithms predicts outcomes in hematopoietic stem cell transplantation
}

\author{
Jun Zou, ${ }^{1 *}$ Piyanuch Kongtim, ${ }^{2 *}$ Betül Oran, ${ }^{3}$ Vasilis Kosmoliaptsis, ${ }^{4}$ Yudith \\ Carmazzi, ${ }^{1}$ Junsheng Ma, ${ }^{5}$ Liang Li, ${ }^{5}$ Gabriela Rondon, ${ }^{3}$ Samer Srour, ${ }^{3}$ Hannah C. \\ Copley, ${ }^{4}$ David Partlow, ${ }^{1}$ Stefan 0. Ciurea, ${ }^{2}$ Uri Greenbaum, ${ }^{3}$ Qing Ma, ${ }^{3}$ Elizabeth \\ J. Shpall, ${ }^{3}$ Richard E. Champlin ${ }^{3 *}$ and Kai $\mathrm{Cao}^{1 \#}$
}

Haematologica 2022

Volume 107(4):844-856

\section{Correspondence:}

JUN ZOU

jzou@mdanderson.org

Received: April 14, 2021.

Accepted: July 9, 2021.

Pre-published: August 26, 2021.

https://doi.org/10.3324/haematol.2021.278993

(C)2022 Ferrata Storti Foundation

Material published in Haematologica is covered by copyright. All rights are reserved to the Ferrata Storti Foundation. Use of published material is allowed under the following terms and conditions:

https://creativecommons.org/licenses/by-nc/4.0/legalcode. Copies of published material are allowed for personal or internal use. Sharing published material for non-commercial purposes is subject to the following conditions:

https://creativecommons. org/licenses/by-nc/4.0/legalcode, sect. 3. Reproducing and sharing published material for commercial purposes is not allowed without permission in writing from the publisher.
${ }^{1}$ Department of Laboratory Medicine, The University of Texas MD Anderson Cancer Center, Houston, TX, USA; ${ }^{2}$ Division of Hematology/Oncology, Department of Medicine, Chao Family Comprehensive Cancer Center, University of California, Irvine, CA, USA; ${ }^{3}$ Department of Stem Cell Transplantation and Cellular Therapy, The University of Texas MD Anderson Cancer Center, Houston, TX, USA; ${ }^{4}$ Department of Surgery, University of Cambridge, and NIHR Cambridge Biomedical Research Centre, Cambridge, UK and ${ }^{5}$ Department of Biostatistics, The University of Texas MD Anderson Cancer Center, Houston, TX, USA

*JZ and PK contributed equally as co-first authors.

"REC and KC contributed equally as co-senior authors.

\section{ABSTRACT}

$\mathrm{H}$ LA-DPB1 mismatches between donor and recipient are commonly seen in allogeneic hematopoietic stem cell transplantation from an unrelated donor. HLA-DPB1 mismatch, conventionally determined by the similarity of the T-cell epitope (TCE), is associated with an increased risk of acute graft-versus-host disease (GVHD) and a decreased risk of disease relapse. We investigated the clinical impact of HLA-DPB1 molecular mismatch quantified by mismatched eplets (ME) and the Predicted Indirectly Recognizable HLA Epitopes Score (PS) in a cohort of 1,514 patients receiving hematopoietic stem cell transplants from unrelated donors matched at HLA-A, -B, -C, -DRB1/3/4/5, and DOB1 loci. HLA-DPB1 alloimmunity in the graft-versus-host direction, determined by high graft-versus-host ME/PS, was associated with a reduced risk of relapse (hazard ratio $[\mathrm{HR}]=0.83, P=0.05$ for $\mathrm{ME}$ ) and increased risk of grade $2-4$ acute GVHD ( $\mathrm{HR}=1.44, P<0.001$ for $\mathrm{ME}$ ), whereas high host-versus-graft ME/PS was only associated with an increased risk of grade $2-4$ acute GVHD ( $\mathrm{HR}=1.26, P=0.004$ for $\mathrm{ME}$ ). Notably, in the permissive mismatch subgroup classified by TCE grouping, high host-versus-graft ME/PS was associated with an increased risk of relapse ( $\mathrm{HR}=1.36, P=0.026$ for $\mathrm{ME})$ and grade 2-4 acute GVHD ( $\mathrm{HR}=1.43, P=0.003$ for PS-II). Decision curve analysis showed that graftversus-host $\mathrm{ME}$ outperformed other models and provided the best clinical net benefit for the modification of acute GVHD prophylaxis regimens in patients with a high risk of developing clinically significant acute GVHD. In conclusion, molecular assessment of HLA-DPB1 mismatch enables separate prediction of host-versus-graft or graft-versus-host alloresponse quantitatively and allows further refinement of HLA-DPB1 permissiveness as defined by conventional TCE grouping.

\section{Introduction}

Currently, allogeneic hematopoietic stem cell transplantation (HSCT) is the only curative therapy for many hematologic malignancies. Although modern immunosuppressive therapy and transplant interventions have significantly improved non-relapse mortality (NRM) over years, ${ }^{1}$ as a major complication after HSCT, acute graft-versus-host disease (GVHD) occurs in 20 to $80 \%$ of recipients with $15 \%$ mortality. ${ }^{2}$ It is well established that patients who undergo allogeneic HSCT 
from an HLA-mismatched unrelated donor are more likely to have a higher incidence of acute GVHD and suboptimal clinical outcomes. ${ }^{3-5}$ Among patients who have received HLA-A, $-\mathrm{B},-\mathrm{C},-\mathrm{DRB} 1$, and $-\mathrm{DOB} 1$ matched $(10 / 10)$ grafts from unrelated donors, the disparity between the donor and recipient at the HLA-DPB1 locus is associated with an increased risk of GVHD but is counterbalanced by a reduced risk of relapse. ${ }^{6,7}$

Given the weak linkage disequilibrium between the DP locus and DR/DQ loci, mismatching at the HLA-DPB1 locus is observed in about $75-90 \%$ of transplants from unrelated donors regardless of matching at other HLA loci. ${ }^{7-10}$ Pioneering studies have classified HLA-DPB1 mismatches as permissive or nonpermissive using the functional toxicity assay and by analyzing the similarity of $\mathrm{T}$ cell epitopes (TCE). ${ }^{11,12}$ The initial experimental hypothesis has been confirmed clinically and translated into a donor selection algorithm; permissive HLA-DPB1 mismatches are associated with elicited alloreactivity resulting in a beneficial graft-versus-leukemia (GVL) effect with clinically tolerable GVHD. ${ }^{13,14}$ This approach has significantly expanded the likelihood of finding suitable unrelated donors and reduced the risks of mortality by avoiding donors with nonpermissive mismatches.,15,16 Although the TCE model assigns permissiveness based on T-cell alloreactivity within the same or from different immunogenicity groups, ${ }^{11}$ another partially overlapping model predicts HLA-DPB1 immunogenicity with similar success by analyzing expression levels of the specific HLA-DPB1 allele. . $^{17,18}$

Modern HLA molecular matching methods may open new avenues for alloimmune risk assessment and help to quantitatively refine the traditional TCE grouping. Additionally, the different direction of HLA-DPB1 nonpermissive mismatches defined by the TCE model, i.e., either in the host-versus-graft (HVG) or graft-versus-host (GVH) direction, appears to have a similar impact on the risk of GVHD and mortality in HSCT from unrelated donors. ${ }^{7,8,16}$ Although the underlying mechanism of nonpermissive mismatch in the HVG direction remains unclear, recent compelling evidence showed that peripheral host $\mathrm{T}$ cells present in the skin and gut are primed by donor-derived antigen-presenting cells and contribute to the development of GVHD. ${ }^{19-21}$ Computational prediction methods could separately assess immunogenicity from a donor's or recipient's perspective in a quantitative manner, which might shed light on the alloreactive mechanisms that mediate GVHD risk and the GVL effect in HSCT from HLA-DPB1 mismatched donors.

HLAMatchmaker, one of the best-studied molecular matching strategies, compares eplets, which are the key structural component of epitopes, between the donor and recipient. The amount of mismatched eplets (ME) between donor and recipient has been shown to correlate with the level of immune response and is associated with clinical outcomes in patients who have undergone haploidentical HSCT. ${ }^{22}$ As HLAMatchmaker focuses mainly on surface-accessible positions, TCE that are derived from polymorphisms on the non-exposed region of HLA molecules could be overlooked. ${ }^{23,24}$ Alloreactivity in transplantation is critically dependent on T-cell responses via the indirect recognition pathway in which polymorphic HLA-derived peptides are presented to T cells. Although various approaches have been described to predict TCE through the indirect recognition pathway, Predicted Indirectly Recognizable HLA Epitopes (PIRCHE), with PIRCHE score (PS)-I representing CD $8^{+}$T-cell alloreactivity and PS-II representing $\mathrm{CD}^{+} \mathrm{T}$-cell alloreactivity, is widely and successfully used for this purpose. ${ }^{25}$

In the present study, we sought to comprehensively validate the molecular mismatch algorithms in predicting the risks associated with HLA-DPB1 mismatches in a relatively large cohort of patients with malignant disease who underwent HSCT from unrelated donors. Furthermore, we hypothesized that in silico quantification could refine the current definition of TCE grouping, especially in the permissive or nonpermissive mismatch subgroups, given that significantly different T-cell cross-reactivities are seen in various HLA-DPB1 alleles within the same subgroup. ${ }^{11}$

\section{Methods}

\section{Patients and transplant characteristics}

Our cohort included consecutively treated patients with hematologic malignancies who were 18 years of age or older and underwent allogeneic HSCT at The University of Texas MD Anderson Cancer Center (UTMDACC) between June 2005 and December 2018. All patients in our analysis received HSCT from an HLA-A, -B, -C, DRB1, -DQB1, -DRB3/4/5 matched unrelated donor to minimize the confounding alloreactivity caused by HLA mismatch from other loci. Clinical and laboratory data were collected from electronic medical records.

All patients provided written informed consent for HSCT in accordance with the Declaration of Helsinki. A retrospective data review protocol and a waiver of informed consent were approved by the UTMDACC Institutional Review Board.

\section{HLA typing and ME and PS analyses}

Patients included in the study had donor and recipient HLA typing performed at the HLA-A, -B, -C, DRB1, -DRB3/4/5, $\mathrm{DQB1}$, and -DPB1 loci using sequence-based typing methods at high resolution. ${ }^{26} \mathrm{ME}$ load at the HLA-DPB1 locus was measured using the HLAMatchmaker module incorporated in HLA Fusion software v4.3, which identifies theoretically predicted eplets based on crystallized HLA molecule models ${ }^{27}$ and identifies ME by comparing donor and recipient eplets. The analyses were performed separately in both the GVH and HVG directions. ${ }^{22}$ Eplet repertoires are listed in the HLA Epitope Registry (http://www.epitopes.net/downloads.html). The PS for mismatched HLA-DPB1 in the GVH direction was calculated using the HSCT module from the PIRCHE online matching service (http://www.pirche.com/pirche/\#/). The PS for mismatched HLADPB1 in the HVG direction was calculated by inverting the patient and donor in the input fields using the same HSCT module.

\section{HLA-DPB1 permissiveness defined by the TCE model}

HLA-DPB1 mismatches between the donor and the recipient were classified into permissive and nonpermissive mismatches according to TCE algorithms (version 2.0) on the IPD-IMGT/HLA website (https://www.ebi.ac.uk/ipd/imgt/hla/dpb.html). ${ }^{28}$ As previously described, ${ }^{26}$ the direction of HLA-DPB1 mismatch, either in the GVH or HVG direction, was assigned. Transplants were therefore classified into four groups: (i) HLA-DPB1 matched, (ii) permissive mismatched, (iii) nonpermissive mismatched in the HVG direction, and (iv) nonpermissive mismatched in the GVH direction. 


\section{Statistical analysis}

The primary outcome was acute GVHD and secondary outcomes were overall survival, progression-free survival, relapse, NRM, and neutrophil engraftment.

Univariate and multivariable Cox proportional hazards regression was used to determine the impact of baseline characteristics, PS, ME, and HLA-DPB1 matching on survival outcomes, while univariate and multivariable sub-distributional hazards regression was used to analyze cumulative incidence outcomes, including relapse, NRM, acute GVHD, and engraftment. All regression models were tested for proportional hazards assumption and interaction terms. Each PS, ME, and HLA-DPB1 match group with a $P$ value $<0.1$ in the univariate analysis was analyzed in separate multivariable regression models adjusted for significant baseline characteristics. PS and ME were analyzed as both continuous variables and categorical variables (low versus high), and they were analyzed only as categorical variables in multivariable analyses. To determine the optimal cutoff for low versus high $\mathrm{PS}$ and $\mathrm{ME}$ groups, the concordance probabilities of PS and ME for acute GVHD prediction were tested at the $25^{\text {th }}$, $50^{\text {th }}$, and $75^{\text {th }}$ percentile cutoffs. The cutoffs at the $50^{\text {th }}$ percentile were selected for the analysis to maximize the concordance probability.

The discrimination power of the TCE, ME, and PS models on acute GVHD was compared using the Harrell C-concordance index. A decision-curve analysis ${ }^{29,30}$ was performed to assess the net clinical benefit of all models in deciding on GVHD regimen modification

Outcome definitions and details of the statistical analysis are described in the Online Supplementary Material.

\section{Results}

\section{Patients' characteristics and HLA-DPB1 matching status defined by TCE and in silico methods}

The analysis included 1,514 patients with a median age of 56 years (range, 18-79). The characteristics of the patients and their transplants are listed in Table 1. The majority of patients received a peripheral blood graft $(62 \%)$ and GVHD prophylaxis with tacrolimus and mycophenolate (83\%). Seventy-four percent of patients received anti-thymocyte globulin as a part of GVHD prophylaxis. The variables that were significantly different between subgroups were bone marrow stem cell source (with $29 \%$ in the GVH nonpermissive group versus $37.6 \%$ in the whole group) and the year of HSCT. The number of transplants with nonpermissive mismatch was significantly reduced in recent years (2014-2018) compared to the previous years $(27.6 \%$ versus $36.5 \%$, respectively), likely due to the awareness of the adverse effect of nonpermissive mismatch.

HLA-DPB1 permissive mismatch was present in $43.0 \%$ of patients, and nonpermissive HLA-DPB1 mismatches in the GVH and HVG directions were noted in $17.7 \%$ and $15.1 \%$ of patients, respectively. The median follow-up duration in 695 surviving patients was 57.1 months (range, 3.4-148.4).

ME, PS-I, and PS-II were quantified in both HVG and GVH directions (Table 1). High concordances between the functional TCE grouping and in silico methods were noted. The median ME, PS-I, and PS-II values in the GVH direction in the GVH nonpermissive mismatch group were significantly higher than the corresponding values in the HVG nonpermissive mismatch group and in the permissive mismatch group. Likewise, the median ME, PS-I, and PS-II values in the HVG direction were considerably higher in the HVG nonpermissive mismatch group than in the GVH nonpermissive mismatch and permissive mismatch groups.

No or weakly positive correlations were seen between GVH and HVG ME, PS-I, and PS-II values, indicating that ME/PS from the donor perspective were different from ME/PS from the recipient perspective, whereas positive correlations were observed between PS-I and PS-II values and between ME and PS values in the same direction (GVH or HVG) (Online Supplementary Figure S1). The number of patients in the low and high PS and ME groups and TCE model are summarized in Online Supplementary Tables S1 and S2.

\section{Impact of HLA-DPB1 matching status defined by TCE, ME, and PS on post-transplant outcomes}

In the entire cohort, molecular mismatches in the GVH direction were associated with a reduced risk of relapse and increased risk of GVHD and NRM, whereas mismatch in the HVG direction was associated only with increased risk of GVHD without relapse protection.

Results from multivariable analyses showed that HLADPB1 mismatches by TCE grouping, ME, PS-I, and PS-II in both the GVH and HVG directions were strongly associated with an increased risk of clinically significant acute GVHD after adjustment for significant baseline characteristics (Figure 1A). Using conventional TCE grouping, compared with the HLA-DPB1 matched group, those with permissive mismatch, GVH nonpermissive mismatch, and HVG nonpermissive mismatch had an increased risk of grade 2-4 acute GVHD (permissive: hazard ratio $[\mathrm{HR}]=1.42,95 \%$ confidence interval $[95 \% \mathrm{CI}$ : 1.15-1.76, $P=0.001$; GVH nonpermissive: $\mathrm{HR}=1.99,95 \%$ CI: 1.55-2.55, $P<0.001$; HVG nonpermissive: $\mathrm{HR}=1.80$, 95\% CI: 1.38-2.35, $P<0.001)$.

Using the median cutoff of ME, the risk of grade 2-4 acute GVHD was 1.44 (95\% CI: $1.23-1.68, P<0.001)$ and 1.26 (95\% CI: $1.08-1.48, P=0.004)$ times higher in those with high ME in the GVH and HVG direction, respectively, than in those with low $M E$ in the same direction. Similarly, having a high PS in the GVH direction was associated with an increased risk of grade 2-4 acute GVHD (PS-I: HR $=1.39,95 \%$ CI: 1.19-1.63, $P<0.001$; PS-II: $\mathrm{HR}=1.40,95 \%$ CI: 1.19-1.64, $P<0.001)$. Having a high PS in the HVG direction was also associated with an increased risk of grade 2-4 acute GVHD (PS-I: HR=1.32, 95\% CI: $1.12-1.54, P=0.001$; PS-II: $\mathrm{HR}=1.24,95 \%$ CI: 1.05-1.45, $P=0.009$ ).

The associations of ME, PS-I, and PS-II in the GVH direction with grade 2-4 acute GVHD risk were independent of the associations of ME, PS-I, and PS-II in the HVG direction with grade 2-4 acute GVHD. However, higher risks of grade 2-4 acute GVHD were seen in patients who had high ME, PS-I, or PS-II in both the GVH and HVG directions than in those with low ME, PS-I, or PS-II in both directions (Figure 1A, Online Supplementary Figure S2A).

For NRM, HLA-DPB1 nonpermissive mismatch in either the GVH direction (HR=1.67, 95\% CI: 1.24-2.27, $P=0.001)$ or HVG direction (HR $=1.46,95 \%$ CI: $1.05-2.03$, $P=0.025)$ was associated with a significantly increased risk of NRM compared with that in the matched group, whereas no association was seen between NRM and permissive mismatch status. The strong association of high 
Table 1. Clinical characteristics of patients who underwent hematopoietic stem cell transplantation from unrelated donors.

\begin{tabular}{|c|c|c|c|c|c|c|}
\hline & & & FIA-DPB & match by TCE grouping & & \\
\hline Characteristic & $\begin{array}{c}\text { Entire cohort, } \\
\qquad n=1514\end{array}$ & $\begin{array}{l}\text { Match, } \\
n=366\end{array}$ & $\begin{array}{l}\text { Permissive } \\
\text { mismatch, } \\
n=651\end{array}$ & $\begin{array}{c}\text { GVH nonpermissive } \\
\text { mismatch, } \\
n=269\end{array}$ & $\begin{array}{l}\text { HVG nonpermissive } \\
\text { mismatch, } \\
n=228\end{array}$ & P \\
\hline Median age in years (range) & $56(18-79)$ & $55(18-76)$ & $56(18-76)$ & $56(20-77)$ & $57(20-79)$ & 0.972 \\
\hline Age > 50 years, n (\%) & $991(65.5)$ & $237(64.8)$ & $437(67.1)$ & $172(63.9)$ & $145(63.6)$ & 0.673 \\
\hline Donor age in years (range) & $30(18-71)$ & $30(18-63)$ & $30(18-58)$ & $30(18-59)$ & $29(19-71)$ & 0.387 \\
\hline Donor age $>40$ years, $n(\%)$ & $288(19.0)$ & $59(16.1)$ & $122(18.8)$ & $61(22.7)$ & $46(20.2)$ & 0.207 \\
\hline Female, n (\%) & $614(40.6)$ & $141(38.5)$ & $259(39.8)$ & $120(44.6)$ & $94(41.2)$ & 0.447 \\
\hline $\begin{array}{l}\text { Donor-recipient sex combination, } \mathrm{n}(\%) \\
\text { Female to female } \\
\text { Female to male } \\
\text { Male to female } \\
\text { Male to male }\end{array}$ & $\begin{array}{l}178(11.8) \\
211(13.9) \\
436(28.8) \\
689(45.5)\end{array}$ & $\begin{array}{l}42(11.5) \\
48(13.1) \\
99(27.0) \\
177(48.4)\end{array}$ & $\begin{array}{l}74(11.4) \\
97(14.9) \\
185(28.4) \\
295(45.3)\end{array}$ & $\begin{array}{l}35(13.0) \\
42(15.6) \\
85(31.6) \\
107(39.8)\end{array}$ & $\begin{array}{l}27(11.8) \\
24(10.5) \\
67(29.4) \\
110(48.3)\end{array}$ & 0.566 \\
\hline $\begin{array}{l}\text { ABO matching, } \mathrm{n}(\%) \\
\text { Match } \\
\text { Minor mismatch } \\
\text { Major mismatch } \\
\text { Bidirectional mismatch }\end{array}$ & $\begin{array}{c}724(47.8) \\
351(23.2) \\
333(22.0) \\
106(7.0)\end{array}$ & $\begin{array}{l}177(48.4) \\
77(21.0) \\
81(22.1) \\
31(8.5)\end{array}$ & $\begin{array}{l}313(48.1) \\
159(24.4) \\
147(22.6) \\
32(4.9)\end{array}$ & $\begin{array}{l}129(48.0) \\
56(20.8) \\
62(23.0) \\
22(8.2)\end{array}$ & $\begin{array}{l}105(46.1) \\
59(25.9) \\
43(18.9) \\
21(9.2)\end{array}$ & 0.259 \\
\hline $\begin{array}{l}\text { Donor-recipient CMV serostatus ( } \mathrm{n}=1510 \\
\text { NR-NR } \\
\text { NR-R } \\
\text { R-NR } \\
\text { R-R }\end{array}$ & $\begin{array}{l}\text { n (\%) } \\
192(12.7) \\
734(48.6) \\
99(6.6) \\
485(32.1)\end{array}$ & $\begin{array}{l}52(14.2) \\
182(49.9) \\
23(6.3) \\
108(29.6)\end{array}$ & $\begin{array}{l}83(12.8) \\
303(46.8) \\
47(7.3) \\
215(33.2)\end{array}$ & $\begin{array}{l}33(12.3) \\
137(50.9) \\
18(6.7) \\
81(30.1)\end{array}$ & $\begin{array}{l}24(10.5) \\
112(49.1) \\
11(4.8) \\
81(35.5)\end{array}$ & 0.725 \\
\hline $\begin{array}{l}\text { Diagnosis, n (\%) } \\
\text { AML/MDS } \\
\text { Other hematologic malignancies }\end{array}$ & $\begin{array}{l}673(44.5) \\
841(55.5)\end{array}$ & $\begin{array}{l}170(46.5) \\
196(53.6)\end{array}$ & $\begin{array}{l}293(45.0) \\
358(55.0)\end{array}$ & $\begin{array}{l}107(39.8) \\
162(60.2)\end{array}$ & $\begin{array}{l}103(45.2) \\
125(54.8)\end{array}$ & 0.376 \\
\hline $\begin{array}{l}\text { DRI, n (\%) } \\
\text { Low } \\
\text { Intermediate } \\
\text { High } \\
\text { Very high }\end{array}$ & $\begin{array}{l}228(15.1) \\
600(39.6) \\
518(34.2) \\
168(11.1)\end{array}$ & $\begin{array}{c}63(17.2) \\
139(38.0) \\
130(35.5) \\
34(9.3)\end{array}$ & $\begin{array}{l}89(13.7) \\
269(41.3) \\
219(33.6) \\
74(11.4)\end{array}$ & $\begin{array}{l}42(15.6) \\
97(36.1) \\
96(35.7) \\
34(12.6)\end{array}$ & $\begin{array}{l}34(14.9) \\
95(41.7) \\
73(32.0) \\
26(11.4)\end{array}$ & 0.710 \\
\hline HCT-CI, median (range) & $3(0-11)$ & $3(0-11)$ & 2 years $(0-11)$ & 3 years $(0-10)$ & 2 years $(0-11)$ & 0.261 \\
\hline HCT-CI $\geq 3$, n (\%) & $766(50.6)$ & $187(51.1)$ & $325(49.9)$ & $150(55.8)$ & $104(45.6)$ & 0.152 \\
\hline Prior AlloHSCT, n (\%) & $36(2.4)$ & $8(2.2)$ & $14(2.2)$ & $8(3.0)$ & $6(2.6)$ & 0.878 \\
\hline Prior AutoHSCT, n (\%) & $120(7.9)$ & $30(8.2)$ & $52(8.0)$ & $21(7.8)$ & $17(7.5)$ & 0.990 \\
\hline $\begin{array}{l}\text { HSCT protocol, } \mathrm{n}(\%) \\
\text { Clinical trial protocol } \\
\text { Standard of care }\end{array}$ & $\begin{array}{l}962(63.5) \\
552(36.5)\end{array}$ & $\begin{array}{l}234(63.9) \\
132(36.1)\end{array}$ & $\begin{array}{l}407(62.5) \\
244(37.5)\end{array}$ & $\begin{array}{l}164(61.0) \\
105(39.0)\end{array}$ & $\begin{array}{l}157(68.9) \\
71(31.1)\end{array}$ & 0.274 \\
\hline $\begin{array}{l}\text { Conditioning regimen intensity, } \mathrm{n}(\%) \\
\text { MA } \\
\text { RIC/NMA }\end{array}$ & $\begin{array}{c}1024(67.6) \\
490(32.4)\end{array}$ & $\begin{array}{l}245(66.9) \\
121(33.1)\end{array}$ & $\begin{array}{l}454(69.7) \\
197(30.3)\end{array}$ & $\begin{array}{l}183(68.0) \\
86(32.0)\end{array}$ & $\begin{array}{l}142(62.3) \\
86(37.7)\end{array}$ & 0.223 \\
\hline $\begin{array}{l}\text { Stem cell source, } n(\%) \\
\text { PB } \\
\text { BM }\end{array}$ & $\begin{array}{l}945(62.4) \\
569(37.6)\end{array}$ & $\begin{array}{l}202(55.2) \\
164(44.8)\end{array}$ & $\begin{array}{l}411(63.1) \\
240(36.9)\end{array}$ & $\begin{array}{l}191(71) \\
78(29)\end{array}$ & $\begin{array}{l}141(61.8) \\
87(38.2)\end{array}$ & 0.001 \\
\hline $\begin{array}{l}\text { GVHD regimen }(\mathrm{n}=1513), \mathrm{n}(\%) \\
\text { Tacrolimus/methotrexate } \\
\text { PTCY } \\
\text { Others }\end{array}$ & $\begin{array}{l}1268(83.8) \\
185(12.2) \\
60(4.0)\end{array}$ & $\begin{array}{l}295(80.6) \\
5515.0 \\
16(4.4)\end{array}$ & $\begin{array}{l}547(84.2) \\
79(12.2) \\
24(3.7)\end{array}$ & $\begin{array}{c}229(85.1) \\
29(10.8) \\
11(4.0)\end{array}$ & $\begin{array}{c}197(86.4) \\
22(9.7) \\
9(4.0)\end{array}$ & 0.540 \\
\hline ATG, n (\%) & $1121(74.0)$ & $266(72.7)$ & $477(73.3)$ & $205(76.2)$ & $173(75.9)$ & 0.657 \\
\hline $\begin{array}{l}\text { Year of HSCT, } n(\%) \\
2005-2009 \\
2010-2013 \\
2014-2018\end{array}$ & $\begin{array}{l}359(23.7) \\
531(35.1) \\
624(41.2)\end{array}$ & $\begin{array}{l}68(18.6) \\
105(28.7) \\
193(52.7)\end{array}$ & $\begin{array}{l}163(25.0) \\
229(35.2) \\
259(39.8)\end{array}$ & $\begin{array}{l}72(26.8) \\
103(38.3) \\
94(34.9)\end{array}$ & $\begin{array}{l}56(24.6) \\
94(41.2) \\
78(34.2)\end{array}$ & $<0.001$ \\
\hline $\begin{array}{l}\text { Quantified ME, PS-I, and PS-II, median (r } \\
\text { GVH DP ME } \\
\text { GVH PS-I } \\
\text { GVH PS-II } \\
\text { HVG DP ME } \\
\text { HVG PS-I } \\
\text { HVG PS-II }\end{array}$ & $\begin{array}{l}4(0-22) \\
0(0-14) \\
2(0-28) \\
4(0-20) \\
0(0-17) \\
1(0-34)\end{array}$ & $\begin{array}{l}0(0-0) \\
0(0-0) \\
0(0-0) \\
0(0-0) \\
0(0-0) \\
0(0-0)\end{array}$ & $\begin{array}{l}5(0-22) \\
1(0-13) \\
3(0-22) \\
5(0-19) \\
1(0-14) \\
3(0-34)\end{array}$ & $\begin{array}{l}9(0-19) \\
3(0-9) \\
8(0-28) \\
5(0-20) \\
0(0-17) \\
1(0-22)\end{array}$ & $\begin{array}{l}5(0-21) \\
1(0-14) \\
2(0-27) \\
9(1-19) \\
3(0-10) \\
8(0-25)\end{array}$ & $\begin{array}{l}<0.001 \\
<0.001 \\
<0.001 \\
<0.001 \\
<0.001 \\
<0.001\end{array}$ \\
\hline
\end{tabular}


Note: Percentages may not add up to 100 because of rounding. $P$ values of categorical variables were from the Fisher exact or $\chi^{2}$ test. $P$ values of continuous variables were from analysis of variance or the Kruskal-Wallis test. There were four missing data points for donor-recipient cytomegalovirus serostatus and one missing data point for the graft-versus-host disease regimen. HSCT: hematopoietic stem cell transplantation; AlloHSCT: allogeneic hematopoietic stem cell transplantation; AutoHSCT: autologous hematopoietic stem cell transplantation;TCE:T-cell epitope; GVH: graft-versus-host;HVG: host-versus-graft;CMV: cytomegalovirus; NR: nonreactive; R: reactive; AML: acute myeloid leukemia; MDS myelodysplastic syndrome; DRI: Disease Risk Index; HCT-CI:Hematopoietic Cell Transplant-Comorbidity Index; MA: myeloablative; RIC: reduced-intensity conditioning; NMA: nonmyeloablative; PB:peripheral blood; BM: bone marrow; GVHD: graft-versus-host disease; PTCY:post-transplant cyclophosphamide;ATG:antithymocyte globulin; DP ME:HLA-DPB1 mismatched eplets; PS-I, Predicted Indirectly Recognizable HLA Epitopes score I; PS-II, Predicted Indirectly Recognizable HLA Epitopes score II.

GVH PS-I and GVH PS-II with grade 2-4 acute GVHD risk resulted in an increased risk of NRM (GVH PS-I: $\mathrm{HR}=1.31$, 95\% CI: 1.07-1.60, $P=0.008$; GVH PS-II: $\mathrm{HR}=1.34,95 \% \mathrm{CI}: 1.10-1.63, P=0.004)$, whereas HVG PSI $(\mathrm{HR}=1.22,95 \% \mathrm{CI}: 1.01-1.49, P=0.041)$, but not HVG PS-II, was associated with an increased risk of NRM, and neither GVH nor HVG ME was significantly associated with NRM. In the analysis of combined groups, NRM risk was highest in those with high GVH and high HVG PS-I ( $\mathrm{HR}=1.48,95 \% \mathrm{CI}: 1.15-1.91, P=0.002)$ and in those with high GVH and high HVG PS-II (HR=1.50, 95\% CI: 1.161.94, $P=0.002$ ) (Figure 1B).
HLA-DPB1 nonpermissive mismatch in the GVH direction was associated with not only an increased risk of acute GVHD but also a reduced risk of relapse $(\mathrm{HR}=0.64$, 95\% CI: $0.47-0.86, P=0.003)$, whereas permissive mismatch and HVG nonpermissive mismatch were not significantly associated with risk of relapse.

Similar results were seen in patients with high GVH ME, PS-I, and PS-II, which were associated with reduced risk of relapse $(\mathrm{ME}: \mathrm{HR}=0.83,95 \% \mathrm{CI}$ : 0.70-0.99, $P=0.05$; PS-I: HR $=0.82,95 \%$ CI: $0.68-0.98, P=0.032$; PS-II: $\mathrm{HR}=0.79$, 95\% CI: 0.66-0.95, $P=0.011$ ), whereas HVG ME, PS-I, and PS-II were not associated with a reduced

A

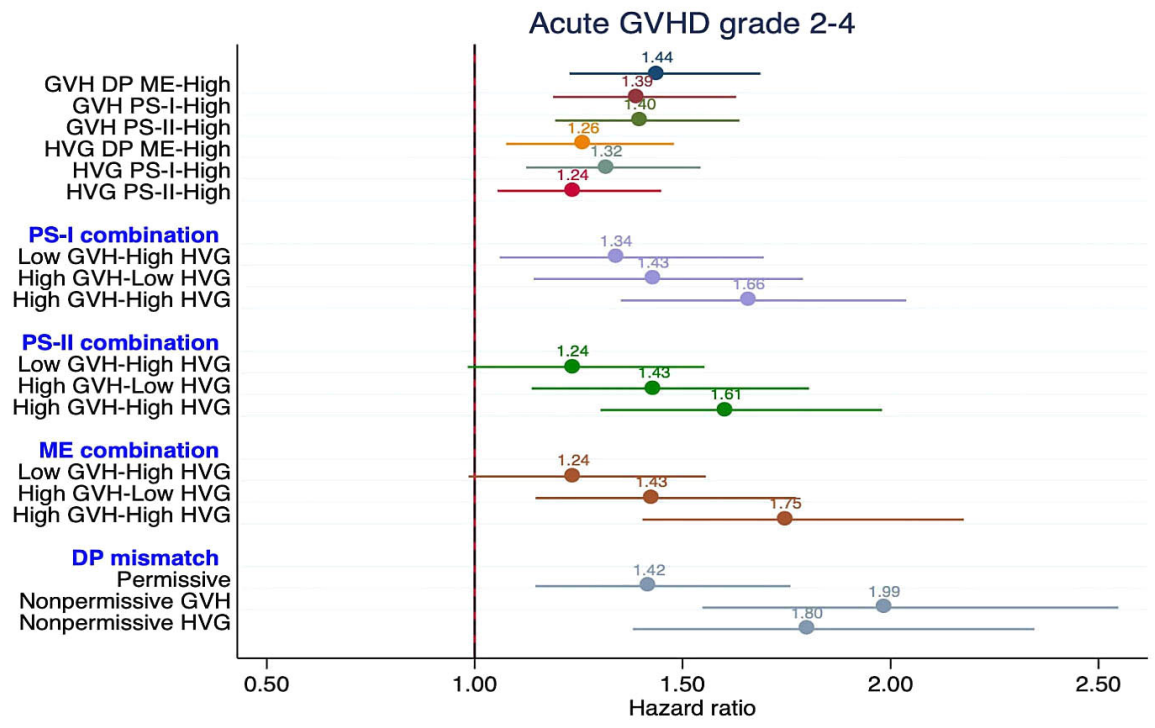

B

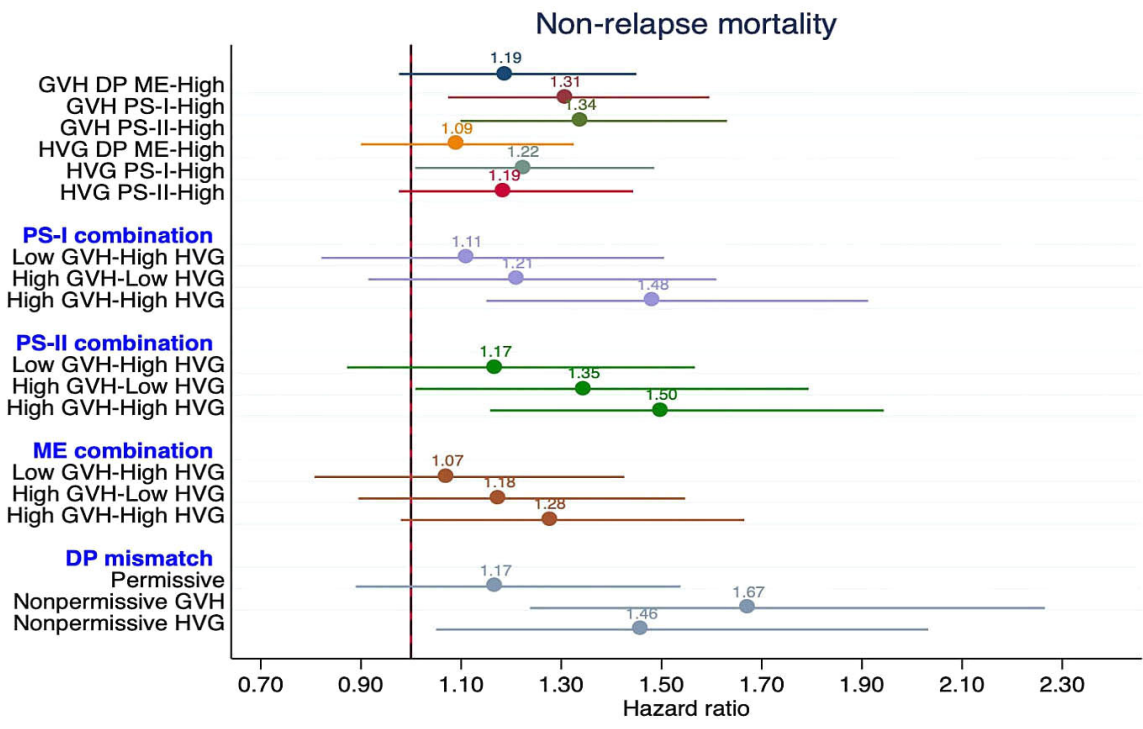

Figure 1. Figure continued on following page. 
C
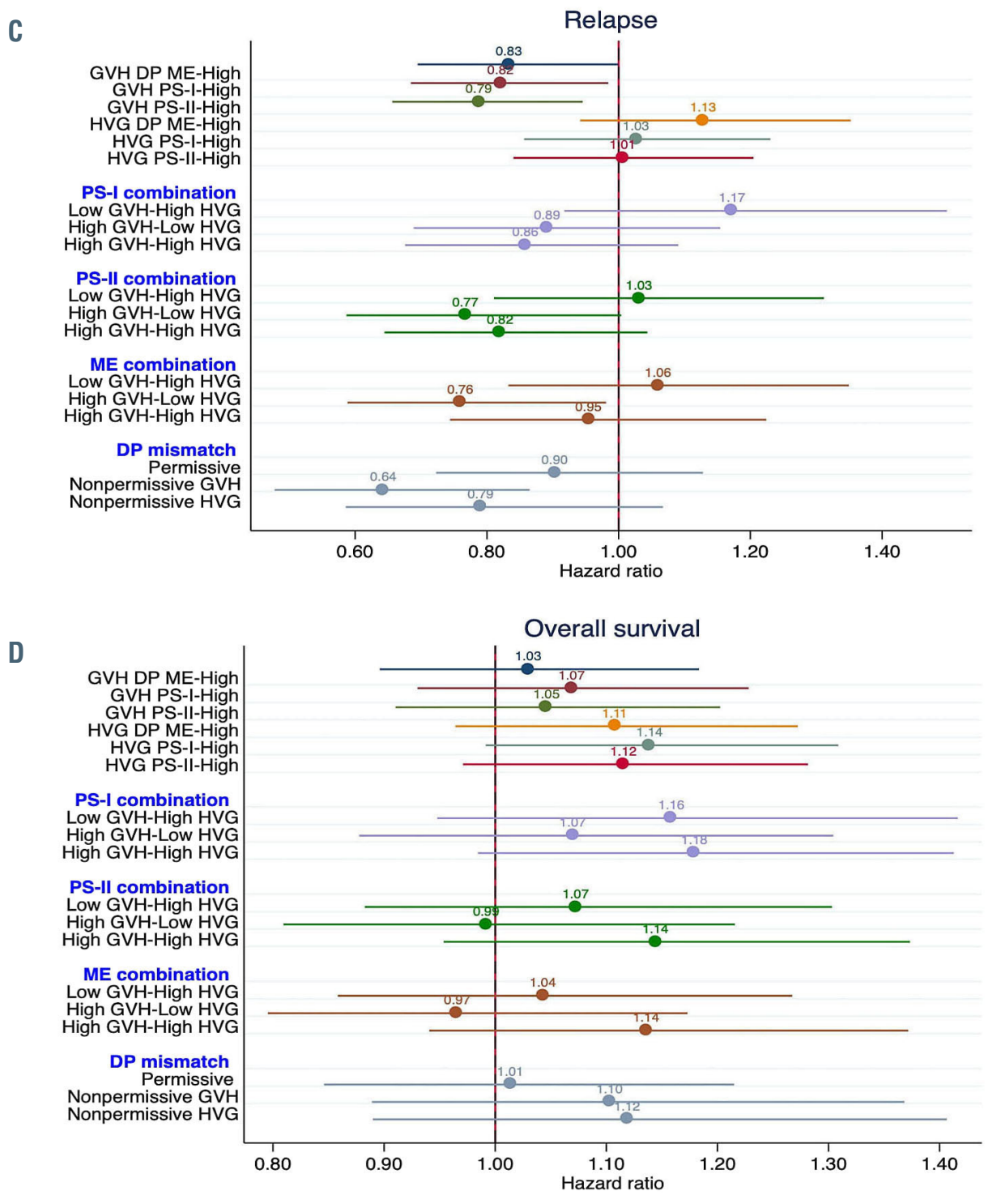

Figure 1. Forest plots showing results from multivariable analyses of the impact of molecular mismatch scores (ME, PS-I, PS-II) and traditional T-cell epitope grouping on outcomes, stratified by the mismatch in the graft-versus-host and host-versus-graft direction. (A) Acute graft-versus-host disease grade 2-4. (B) Non-relapse mortality. (C) Relapse. (D) Overall survival. Dots and bars in the forest plots represent adjusted hazard ratios and 95\% confidence intervals. PS and ME were categorized into low and high groups using the median as a cutoff point. ME: mismatched eplets, PS: Predicted Indirectly Recognizable HLA Epitope score; GVH: graftversus-host: HVG: host-versus-graft; GVHD: graft-versus-host disease.

risk of relapse (Figure 1C). Relapse risk was significantly lower in patients with high GVH ME combined with low HVG ME than in patients with low ME in both directions (Figure 1C, Online Supplementary Figure S2B).

Neither HLA-DPB1 mismatch permissiveness nor molecular mismatches were found to be associated with overall survival (Figure 1D, Online Supplementary Table S3), progression-free survival (Online Supplementary Table S4), or engraftment in the present study cohort.

In the permissive mismatch group, GVH alloimmunity determined by ME and PS was associated with an increased risk of GVHD, whereas HVG alloimmunity determined by ME and PS was associated with an increased risk of relapse and GVHD.

Consistent with the previous report, ${ }^{26}$ permissive mismatch represented the largest subgroup in our cohort of patients who underwent HSCT from unrelated donors. Results from the multivariable analyses showed that the alloimmunity predicted by ME or PS, in either the HVG or the GVH direction, was associated with a trend of increased risk of grade 2-4 acute GVHD (Figure 2A). In particular, HVG PS-II was associated with a significantly increased risk of grade $2-4$ acute GVHD ( $\mathrm{HR}=1.43,95 \%$ CI: $1.13-1.82, P=0.003)$. This finding was further confirmed by our analysis of combined groups, in which a significantly increased risk of grade 2-4 acute GVHD was observed in the group with high ME (Figure 2B) or PS-II in both directions. However, high GVH ME or PS without concurrent HVG alloimmunity was not associated with an increased risk of acute GVHD.

Similar to what we observed in the entire cohort, no anti-leukemia benefit was associated with HVG alloresponse assessed by ME or PS. Moreover, high ME in the HVG direction was associated with an increased risk of relapse in the permissive mismatch group ( $\mathrm{HR}=1.36,95 \%$ $\mathrm{CI}$ : $1.02-1.76, P=0.026$ ) (Figure $2 \mathrm{C}$ ), and this was more 
pronounced in the group with high HVG ME coupled with low alloimmunity in the GVH direction (Figure 2D).

Molecular mismatches assessed by ME or PS were not associated with the risk of NRM, overall survival, or progression-free survival in this permissive mismatch subgroup.

In the GVH nonpermissive mismatch group, ME in the GVH direction was associated with a higher incidence of grade 2-4 acute GVHD, and HVG ME could synergistically contribute to this risk.

Alloimmunity quantified by ME appeared to be more clinically relevant than alloimmunity quantified by PS in the GVH nonpermissive mismatch group. Results from the multivariable analyses showed that high ME in the $\mathrm{GVH}$ direction was associated with an increased risk of grade 2-4 acute GVHD (HR=1.64, 95\% CI: 1.16-2.31, $P=0.005$ ) (Figure $3 \mathrm{~A}$ ). Although HVG ME itself was not associated with the risk of acute GVHD, those with high $\mathrm{ME}$ in both directions had a significantly increased risk of grade $2-4$ acute GVHD (HR=2.82, 95\% CI: 1.41-5.62, $P=0.003$ ) (Figure 3B).

No significant association between the molecular mismatch factors and relapse (Online Supplementary Figure S3), NRM, engraftment, overall survival, or progressionfree survival was identified.

In the HVG nonpermissive mismatch group, ME and PS-I in the GVH direction were associated with worse NRM without an increased risk of GVHD

None of the mismatch factors was associated with the risk of relapse or acute GVHD in the HVG nonpermissive mismatch group with high HVG alloimmunity settings (Online Supplementary Figure $S 4 A, B$ ). Although no association with the risk of acute GVHD was identified, alloimmunity in the GVH direction determined by ME and PS-I was associated with an increased risk of NRM (ME: $\mathrm{HR}=1.90,95 \% \mathrm{CI}: 1.18-3.07, P=0.008$, Figure 4A; PS-I: $\mathrm{HR}=1.60,95 \% \mathrm{CI}: 1.04-2.60, P=0.024$, Figure 4B), indicat-

A

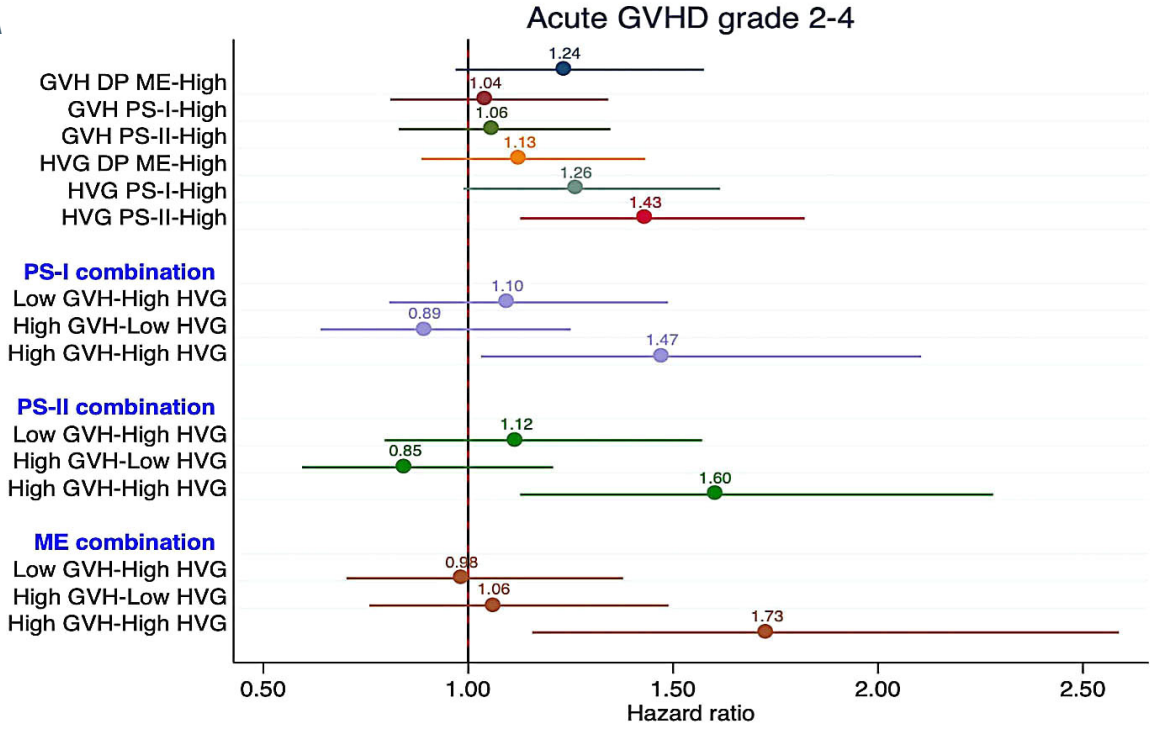

B

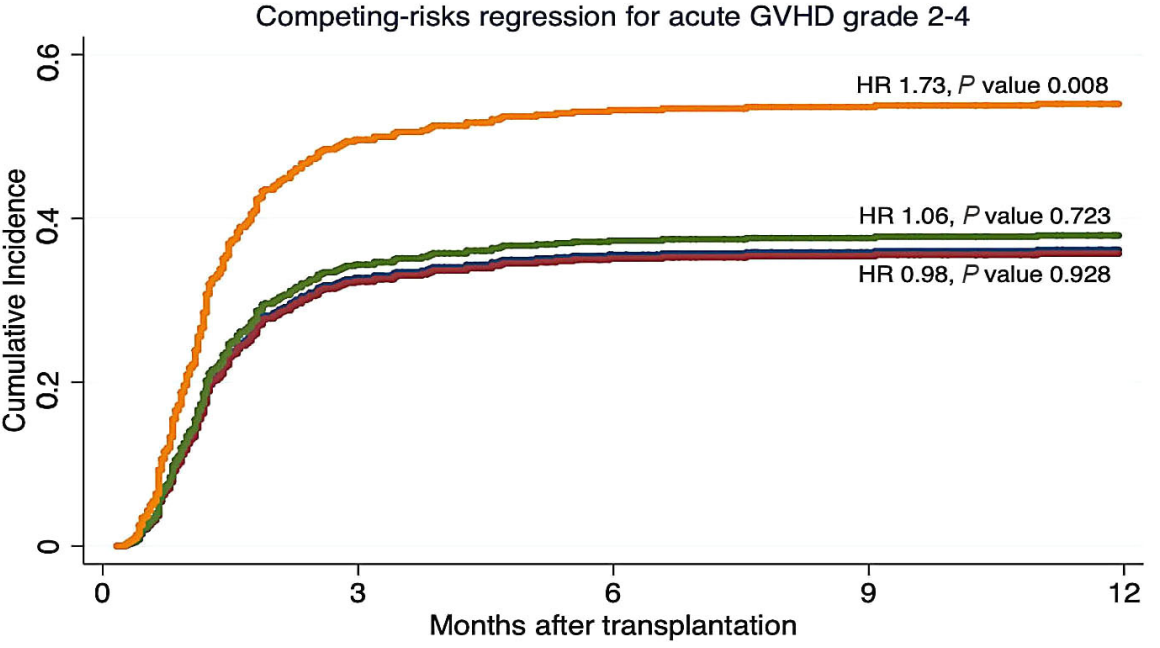

\begin{aligned} \hline Low ME GVH + Low ME HVG & Low ME GVH + High ME HVG \\ High ME GVH + Low ME HVG & High ME GVH + High ME HVG \end{aligned}


C

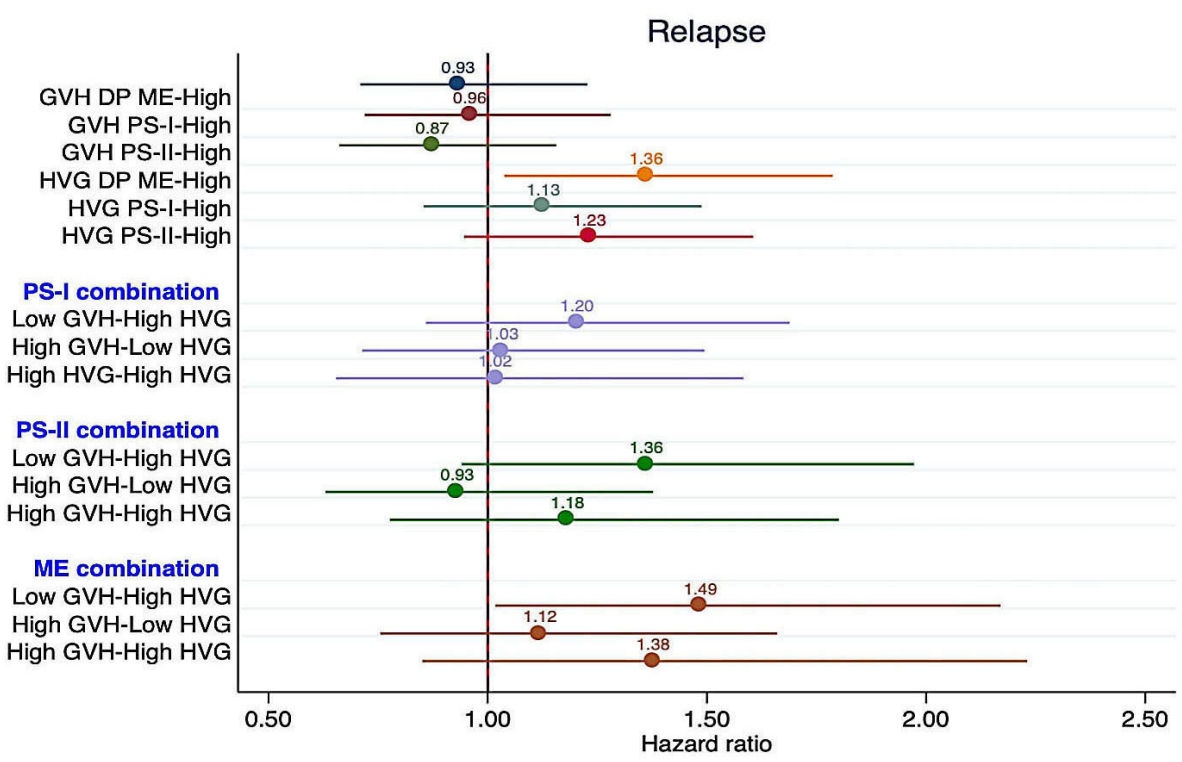

D

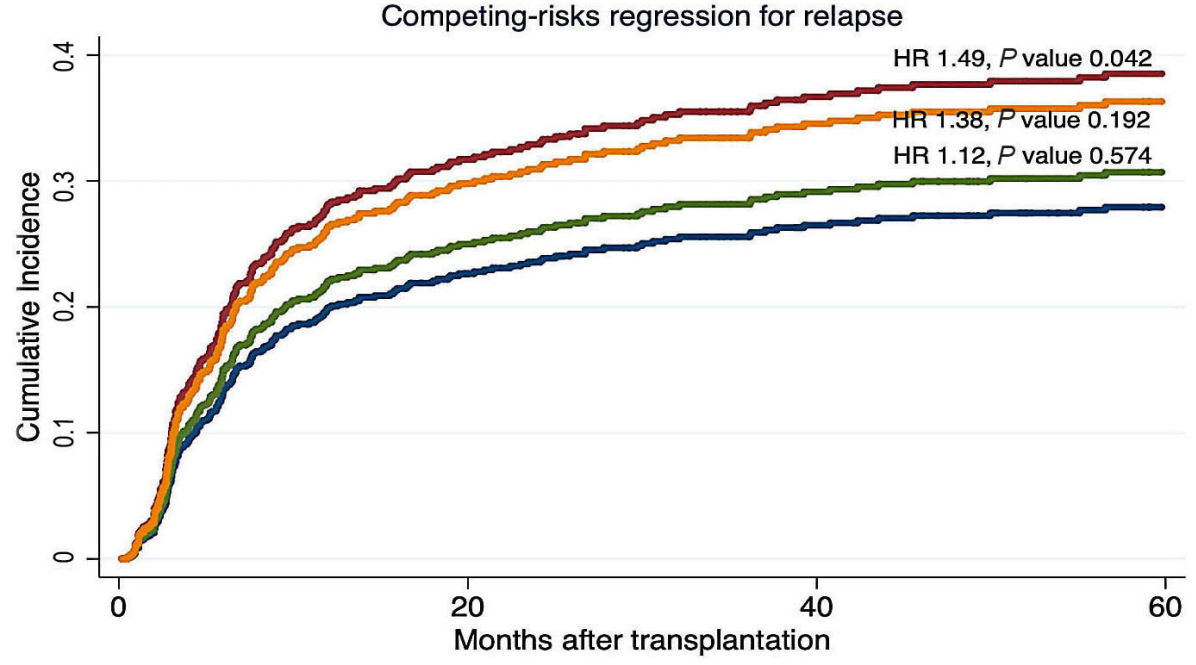

\begin{tabular}{ll}
\hline Low ME GVH + Low ME HVG & Low ME GVH + High ME HVG \\
High ME GVH + Low ME HVG & High ME GVH + High ME HVG
\end{tabular}

Figure 2. Forest plots showing results from the multivariable analyses of the impact of molecular mismatch scores (ME, PS-I, and PS-II) on outcomes in the permissive mismatch group, stratified by the mismatch in the graft-versus-host and host-versus-graft direction. (A) Acute graft-versus-host disease (GVHD) grade 2-4. (B) Adjusted cumulative incidence of acute GVHD grade 2-4. (C) Relapse. (D) Adjusted cumulative incidence of relapse. Dots and bars in the forest plots represent adjusted hazard ratios and 95\% confidence intervals. PS and ME were categorized into low and high groups using the median as a cutoff point. ME: mismatched eplets, PS: Predicted Indirectly Recognizable HLA Epitope score; GVH: graft-versus-host: HVG: host-versus-graft; GVHD: graft-versus-host disease; HR: hazard ratio.

ing that the increased risk of NRM observed here may not be mostly attributed to GVHD. Additionally, a lower incidence of neutrophil engraftment was observed in the group with high $\mathrm{ME}$ in the HVG direction, likely attributable to the alloimmunity towards the graft (HR=0.73, 95\% CI: 0.56-0.96, $P=0.028$ for low GVH ME + high HVG ME).

Predictive performance of the TCE, ME, and PS models

Results from the concordance test showed that the ME in the GVH direction provided better discriminative ability for the prediction of clinically significant acute GVHD with a concordance index of 0.595 compared with other models. The values of the concordance index of GVH PS I, GVH PS II, HVG ME, HVG PS I, HVG PS II, and TCE were
$0.560,0.556,0.545,0.541,0.542$, and 0.566 , respectively.

Moreover, decision curve analysis ${ }^{29}$ was conducted to compare the clinical application of different matching models. We found that ME in the GVH direction outperformed other models, including the conventional TCE model, and provided the best net clinical benefit for the modification of the acute GVHD prophylaxis regimen in patients with a high risk of developing clinically significant acute GVHD (Figure 5).

\section{Discussion}

Relapse and GVHD remain two major causes of morbidity and mortality in patients with hematologic malig- 
nancies undergoing HSCT. It has been accepted that donor T-cell-mediated alloimmune responses are the key mediators of beneficial GVL and adverse GVHD effects. A better understanding of T-cell alloreactivity in patients receiving HSCT would help to minimize the risk of GVHD while still preserving GVL activity. With recent progress in bioinformatics and molecular HLA typing, in silico prediction of immunogenicity has evolved rapidly, and several algorithms with a different focus have been shown to be predictive of outcomes in patients who have undergone HSCT. 22,31

In the present comprehensive study in a cohort of patients with hematologic malignancies, we demonstrat- ed that HLAMatchmaker and PIRCHE can be used to assess histocompatibility in HSCT at the molecular level. Using the decision curve analysis method that incorporates clinical considerations, it was found that $\mathrm{ME}$ in the GVH direction has advantages over other predictive models including the conventional TCE model, in aiding the decision whether or not to modify the acute GVHD prophylaxis regimen. In patients with a high risk of developing clinically significant acute GVHD predicted by high $\mathrm{ME}$ in both GVH and HVG directions, the addition of therapy based on $\mathrm{T}$-cell depletion to the prophylactic regimen may reduce the incidences and intensity of GVHD. Moreover, ME and PS can quantitatively refine the con-

A

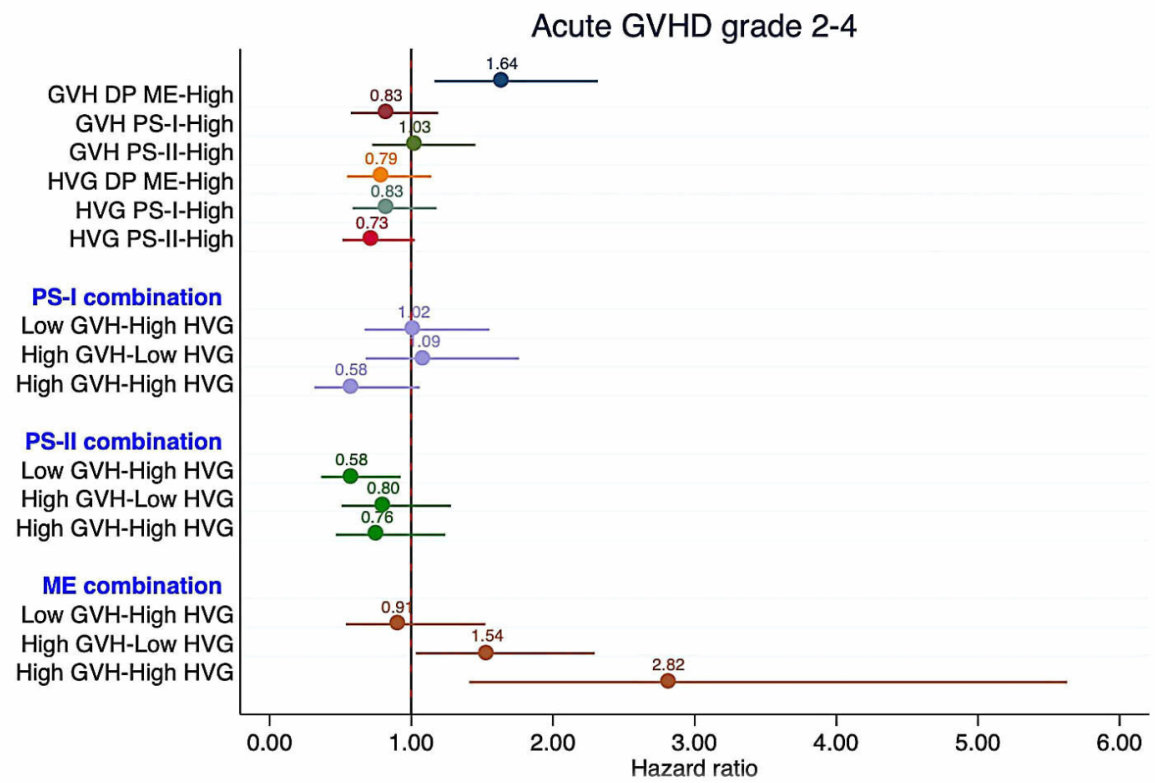

B Competing-risks regression for acute GVHD grade 2-4

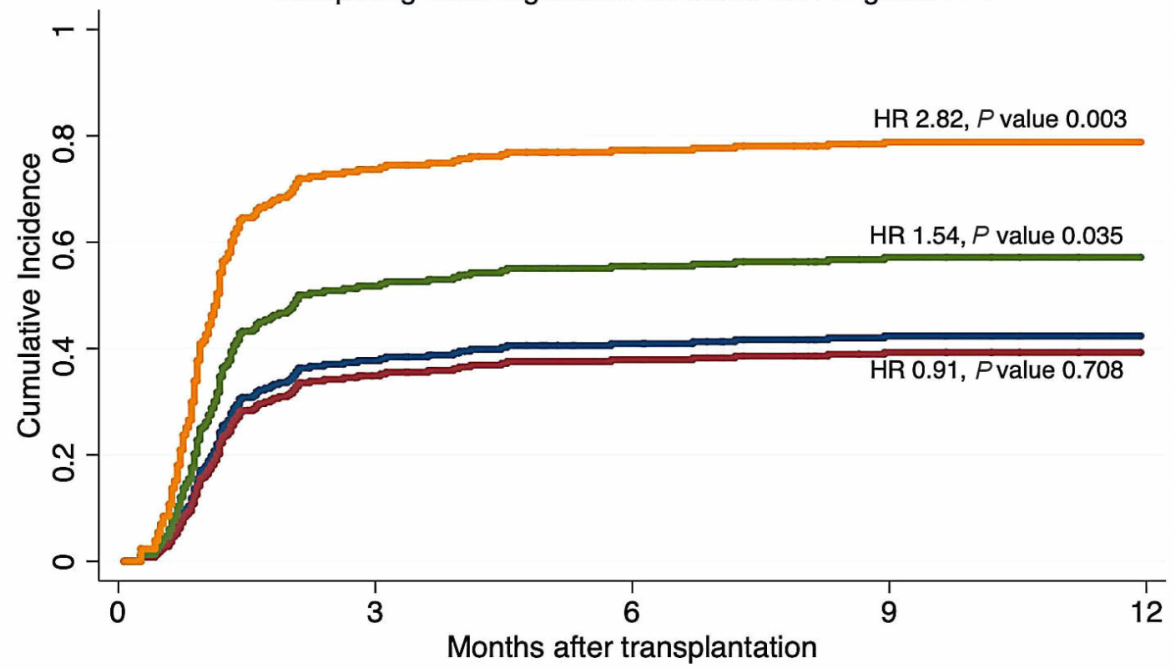

\begin{tabular}{|ll|}
\hline Low ME GVH + Low ME HVG & Low ME GVH + High ME HVG \\
High ME GVH + Low ME HVG & High ME GVH + High ME HVG \\
\hline
\end{tabular}

Figure 3. Forest plots showing results from the multivariable analyses of the impact of molecular mismatch scores (ME, PS-I, PS-II) on outcomes in patients with HLA-DPB1 nonpermissive mismatch in the graft-versus-host (GVH) direction, stratified by ME GVH and host-versus-graft combinations. (A) Acute graft-versus-host disease (GVHD) grade 2-4. (B) Adjusted cumulative incidence of acute GVHD grade 2-4. Dots and bars in the forest plots represent adjusted hazard ratios and 95\% confidence intervals. PS and ME were categorized into low and high groups using the median as a cutoff point. ME: mismatched eplets, PS: Predicted Indirectly Recognizable HLA Epitope score; GVH: graft-versus-host: HVG: host-versus-graft; GVHD: graft-versus-host disease; HR: hazard ratio. 
ventional TCE grouping, so the finding here will aid prioritization of the donors even within the same TCE group.

Using the HLA-DPB1 TCE model, Fleischhauer et al. concluded that mismatches in different directions (HVG versus GVH) did not differ in terms of acute GVHD and mortality risk. ${ }^{32}$ However, bidirectional mismatches seemed to work synergistically and were associated with an increased risk of GVHD. How to reconcile HVG alloimmunity remains unclear, because host $\mathrm{T}$ cells in circulation are believed to be depleted by conditioning regimens during HSCT. Recent studies indicate that peripheral host $T$ cells resident in the skin and gut are stimulated by the mismatched HLA and, as a result, the activated host $\mathrm{T}$ cells secrete higher levels of inflammatory cytokines and contribute to GVHD in addition to graft Tcell immunity. ${ }^{19,21}$ For the first time, we demonstrate that the direction of alloreactivity may be better reflected by ME or PS in different directions. The elicited GVH alloreactivity defined by PS and ME seems to contribute to GVL along with GVHD, whereas HVG alloreactivity is likely to augment GVHD without the anti-leukemia effect. In the HLA-DPB1 permissive mismatch group, the largest subgroup of patients within our cohort, the elicited HVG alloreactivity appears to counteract the antileukemia effect exerted by GVH alloimmunity, discouraging the use of donors with a high load of HVG ME/PS in patients with HLA-DPB1 permissive mismatch. These

A

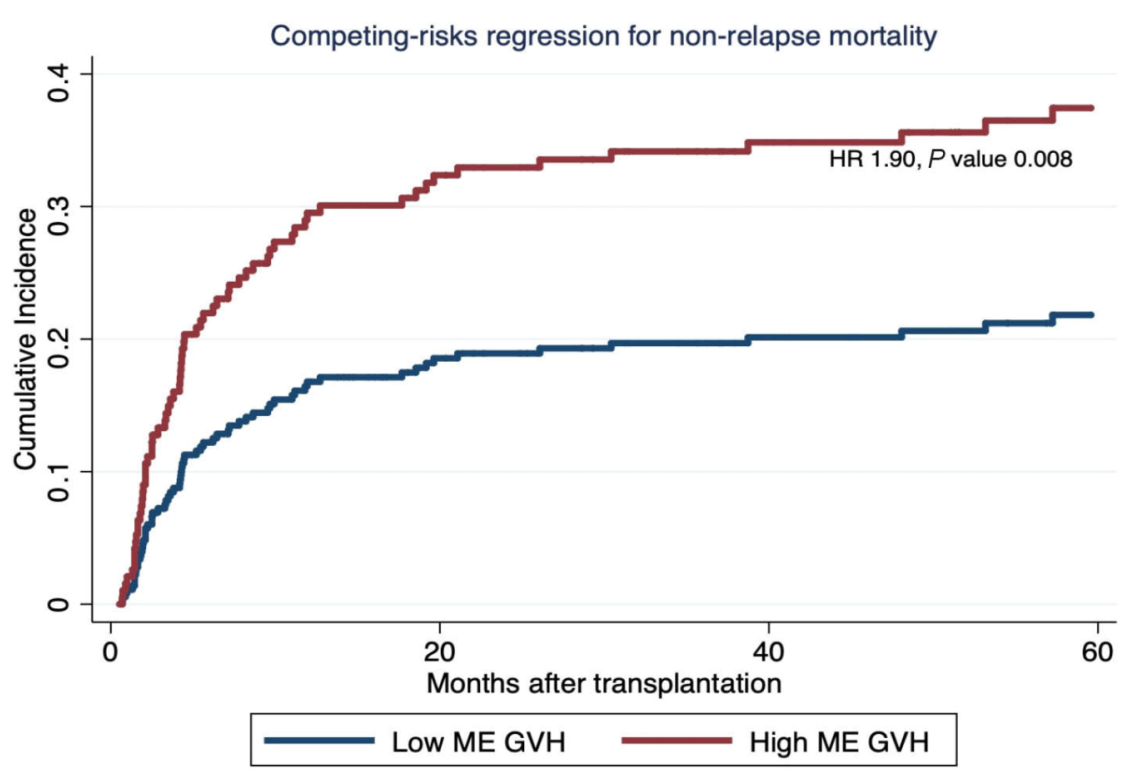

B

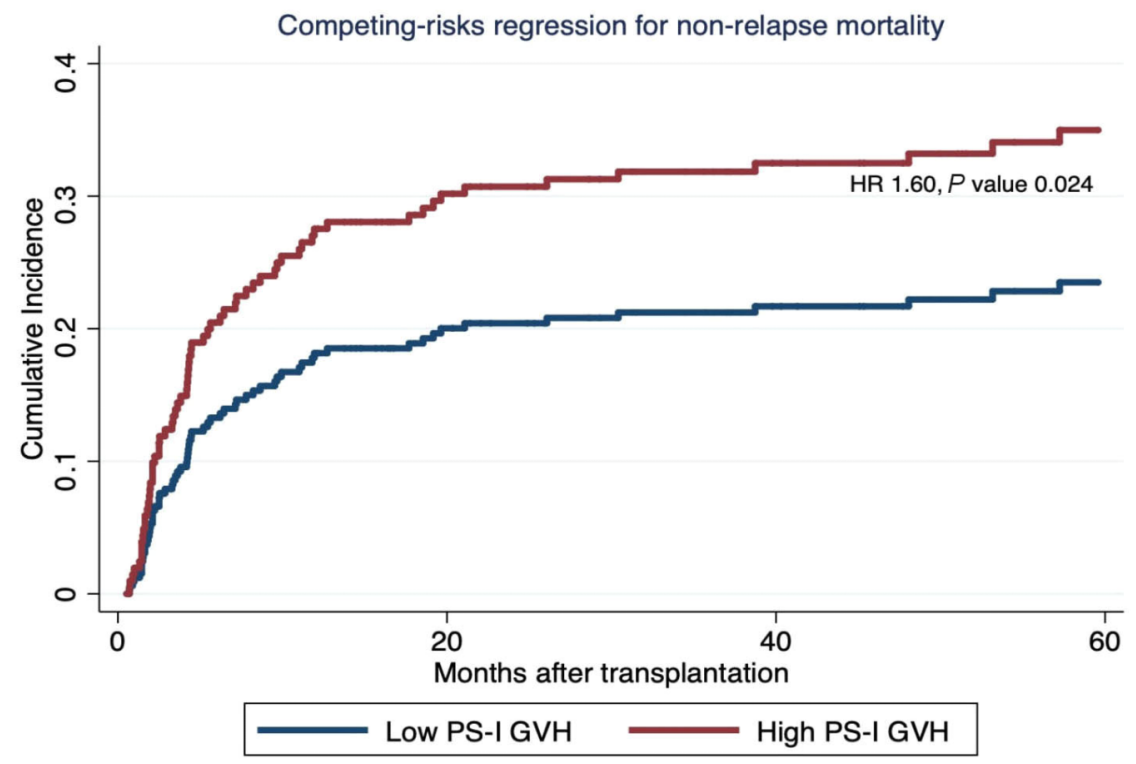

Figure 4. Adjusted cumulative incidence of non-relapse mortality in patients with HLA-DPB1 nonpermissive mismatch in the host-versus-graft direction. (A) Stratified by the number of mismatched eplets (ME) in the graft-versus-host (GVH) direction. (B) Stratified by Predicted Indirectly Recognizable HLA Epitopes score-I (PS-I) in the GVH direction. HR: hazard ratio. 


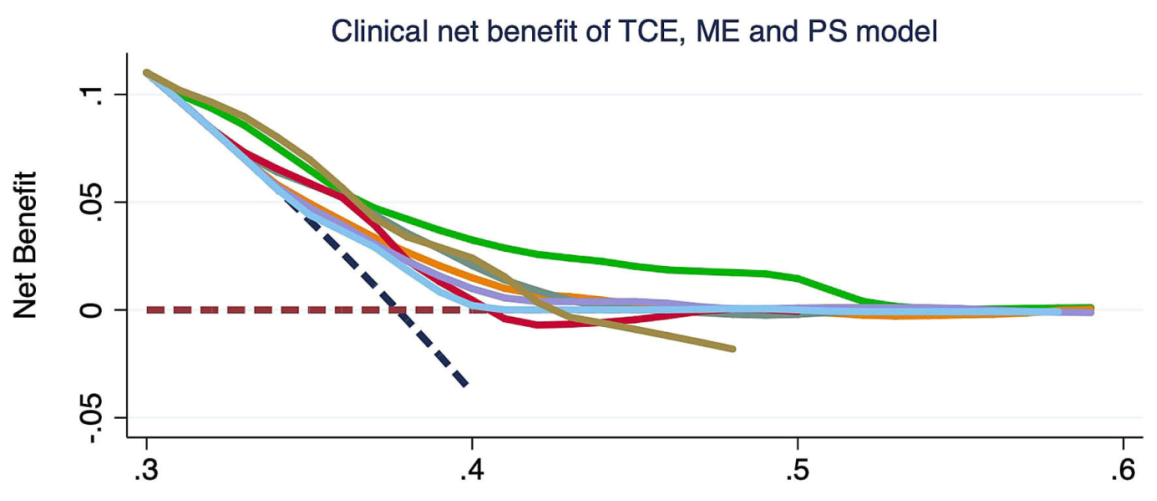

Threshold Probability of aGVHD grade 2-4
Figure 5. The clinical net benefit of the TCE, $\mathrm{ME}$, and PS models in deciding to modify graft-versus-host disease (GVHD) prophylaxis regimen for patients with a high risk of developing clinically significant acute GVHD in comparison with a "treat/modify all" anc "treat/modify none" strategy. Y-axis represents the net clinical benefit (positive values) or risk (negative values) of using model-guided GVHD regimen modification in comparison with no GVHD regimen modification (net clinical benefit $=0$ ). The $X$-axis represents threshold probabilities of acute GVHD grade $2-4$ at 100 days after transplantation. TCE: T-cel epitopes; ME: mismatched eplets, PS Predicted Indirectly Recognizable HLA Epitope score; GVH: graft-versus-host: HVG: host-versus-graft; aGVHD: acute graft-versushost disease.

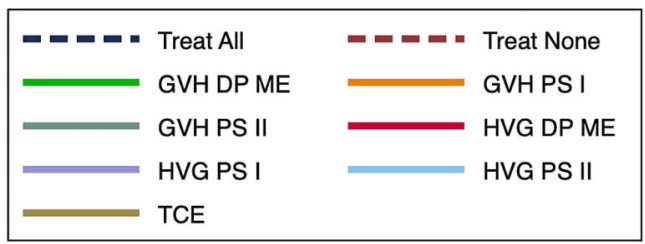

findings not only assist donor selection and risk stratification in HSCT from unrelated donors but also provide valuable insights into the mechanism and process of alloimmunity in this setting.

In agreement with recent studies on DP mismatches using the TCE model ${ }^{33}$ or DP expression model, ${ }^{34}$ associations of the nonpermissive mismatch and overall survival or transplant-related mortality were not found in our cohort. This is perhaps attributable to a high degree of HLA matching degree in the cohort, recent advances in GVHD prophylaxis and reduced incidence of severe GVHD. The majority of our patients received in-vivo Tcell depletion which may lessen the alloresponse derived from DP mismatch and reduce the severity and incidence of acute GVHD. ${ }^{35}$ Additionally, several recent studies documented an improved outcome with post-transplant cyclophosphamide in patients receiving not only haploidentical transplants but also in transplants from matched unrelated donors, ${ }^{36}$ it may be particularly effective for individuals with high ME/PS due to the profound effect of this treatment on GVHD outcomes compared with conventional GVHD prevention regimens. ${ }^{37}$ However, due to the low number of patients who received post-transplant cyclophosphamide in the current study, future large prospective studies are warranted to confirm our hypothesis.

The predictive value of the HLAMatchmaker and PIRCHE algorithms has been demonstrated in HSCT from HLA-mismatched unrelated donors or haploidenti-

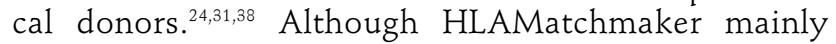
focuses on epitopes directly recognized by B cells, alloreactive T-cell clones that are specific to certain eplets identified by HLAMatchmaker have also been found, ${ }^{39-41}$ suggesting that HLAMatchmaker reveals many polymorphic residues overlapping in both B-cell epitopes and T-cell epitopes. Consistent with a previous study, ${ }^{42}$ we observed a considerable correlation between ME load and PS. However, the disparity determined by ME load appears to be more clinically relevant in our study. Analysis of the topographic location of immunogenic amino acids identified with both methods demonstrated that a significant number of polymorphic amino acids, especially in the $\beta$-sheet and $\alpha$ - 3 domain, were not colocalized. ${ }^{42}$ Therefore, an optimized algorithm that considers both direct and indirect alloresponses would be more predictive of risks or benefits in the context of HSCT with HLA-mismatched donors.

Unlike the TCE and the expression model that has been extensively studied and shown to be clinically relevant for HSCT in several high power studies, ${ }^{7,8,17,18}$ the molecular mismatching algorithms have been primarily studied in the solid organ transplant setting in the assessment of antibody-mediated rejection. The predictive value of ME or PIRCHE was only reported in a few small studies in HSCT settings. ${ }^{38,43-45}$ and further validation is warranted before routine clinical application. The heterogeneity of the cohort and retrospective nature of the current study may have biased our results.

In conclusion, molecular HLA disparity and subsequent alloresponse assessed by in silico methods are useful in the prediction of clinical outcomes. In addition to conventional TCE grouping, additional information provided by $M E$ and $P S$ can be used to refine the permissiveness of HLA-DPB1 mismatches. In the present study, high alloimmunity in both the HVG and GVH directions, revealed by high $\mathrm{PS}$ or $\mathrm{ME}$, is associated with an increased risk of GVHD. Nevertheless, only GVH ME or PS was associated with a reduced risk of relapse. An integrated study in which patients' immune cells are characterized and comprehensively analyzed will provide deeper and better insights into the process of GVH response and the contribution from host $\mathrm{T}$ cells.

\section{Disclosures \\ No conflicts of interest to disclose.}

\section{Contributions}

$J Z, P K, R E C$, and KC designed the study and contributed to collecting and interpreting the data and writing the manuscript; $J Z$ and $P K$ wrote the initial draft of the manuscript; $P K, J M$, and $L L$ contributed to the statistical analysis and interpretation of statistical results and reviewed and approved the manuscript; 
$B O, V K, Y C, S S, H C C, D P, S O C$, and $Q M$ contributed to the data collection and analysis and reviewed and approved the manuscript; GR contributed to data collection and reviewed and approved the manuscript; $B O, S S, U G, E J S$, and REC contributed to the treatment of patients and reviewed, edited, and approved the final version of the manuscript.

\section{Acknowledgments}

The authors would like to thank Kevin Harrell and Dr. JarHow Lee from Thermo Fisher Scientific for their help in eplet analysis for this manuscript. We thank Erica Goodoff, Senior Scientific Editor in the Research Medical Library at The
University of Texas MD Anderson Cancer Center, for editing this article.

\section{Funding}

VK acknowledges funding from an NIHR Fellowship (PDF2016-09-065). This research was partially supported by the Cancer Center Support Grant of MD Anderson (NIH: P30CA016672 to L.L.).

\section{Data-sharing statement}

For data sharing, contact the corresponding author: jzou@mdanderson.org

\section{References}

1. Tanaka Y, Kurosawa S, Tajima K, et al. Analysis of non-relapse mortality and causes of death over 15 years following allogeneic hematopoietic stem cell transplantation. Bone Marrow Ttransplant. 2016;51(4):553559.

2. Pasquini MC, Wang Z, Horowitz MM, Gale RP. 2010 report from the Center for International Blood and Marrow Transplant Research (CIBMTR): current uses and outcomes of hematopoietic cell transplants for blood and bone marrow disorders. Clin Transpl. 2010;87-105.

3. Lee SJ, Klein J, Haagenson M, et al. High-resolution donor-recipient HLA matching contributes to the success of unrelated donor marrow transplantation. Blood. 2007;110 (13):4576-4583

4. Petersdorf EW, Hansen JA, Martin PJ, et al. Major-histocompatibility-complex class I alleles and antigens in hematopoietic-cell transplantation. N Engl J Med. 2001;345 (25):1794-1800

5. Petersdorf EW, Kollman C, Hurley CK, et al. Effect of HLA class II gene disparity on clinical outcome in unrelated donor hematopoietic cell transplantation for chronic myeloid leukemia: the US National Marrow Donor Program experience. Blood. 2001;98(10): 2922-2929.

6. Petersdorf EW, Gooley T, Malkki M, et al. The biological significance of HLA-DP gene variation in haematopoietic cell transplantation. Br J Haematol. 2001;112(4):988-994.

7. Fleischhauer K, Shaw BE, Gooley T, et al. Effect of T-cell-epitope matching at HLADPB1 in recipients of unrelated-donor haemopoietic-cell transplantation: a retrospective study. Lancet Oncol. 2012;13(4): 366-374.

8. Pidala J, Lee SJ, Ahn KW, et al. Nonpermissive HLA-DPB1 mismatch increases mortality after myeloablative unrelated allogeneic hematopoietic cell transplantation. Blood. 2014;124(16):25962606.

9. Varney MD, Lester S, McCluskey J, Gao X, Tait BD. Matching for HLA DPA1 and DPB1 alleles in unrelated bone marrow transplantation. Hum ilmmunol. 1999;60(6):532-538.

10. Hurley CK, Baxter-Lowe LA, Begovich AB, et al. The extent of HLA class II allele level disparity in unrelated bone marrow transplantation: analysis of 1259 National Marrow Donor Program donor-recipient pairs. Bone Marrow Transplant. 2000;25 (4):385-393.

11. Zino E, Frumento G, Marktel S, et al. A Tcell epitope encoded by a subset of HLADPB1 alleles determines nonpermissive mismatches for hematologic stem cell trans- plantation. Blood. 2004;103(4):1417-1424.

12. Fleischhauer K, Locatelli F, Zecca M, et al Graft rejection after unrelated donor hematopoietic stem cell transplantation for thalassemia is associated with nonpermissive HLA-DPB1 disparity in host-versusgraft direction. Blood. 2006;107(7):2984 2992.

13. Fleischhauer K, Shaw BE. HLA-DP in unrelated hematopoietic cell transplantation revisited: challenges and opportunities. Blood. 2017;130(9):1089-1096.

14. Fleischhauer K, Beelen DW. HLA mismatching as a strategy to reduce relapse after alternative donor transplantation. Semin Hematol. 2016;53(2):57-64.

15. Shaw BE, Robinson J, Fleischhauer $\mathrm{K}$ Madrigal JA, Marsh SG. Translating the HLA-DPB1 T-cell epitope-matching algorithm into clinical practice. Bone Marrow Transplant. 2013;48(12):1510-1512

16. Crocchiolo R, Zino E, Vago L, et al. Nonpermissive HLA-DPB1 disparity is a significant independent risk factor for mortality after unrelated hematopoietic stem cell transplantation. Blood. 2009;114(7):14371444.

17. Petersdorf EW, Malkki M, O'Huigin C, et al. High HLA-DP expression and graft-versushost disease. N Engl J Med. 2015;373(7):599 609.

18. Morishima S, Shiina T, Suzuki S, et al Evolutionary basis of HLA-DPB1 alleles affects acute GVHD in unrelated donor stem cell transplantation. Blood. 2018;131(7):808817.

19. Divito SJ, Aasebo AT, Matos TR, et al. Peripheral host $\mathrm{T}$ cells survive hematopoietic stem cell transplantation and promote graft-versus-host disease. J Clin Invest. 2020;130(9):4624-4636.

20. Young JW. Alternative mechanisms that mediate graft-versus-host disease in allogeneic hematopoietic cell transplants. J Clin Invest. 2020;130(9):4532-4535.

21. Jardine L, Cytlak U, Gunawan M, et al. Donor monocyte-derived macrophages promote human acute graft-versus-host disease. J Clin Invest. 2020;130(9):4574-4586.

22. Zou J, Ciurea SO, Kongtim P, et al Molecular disparity in human leukocyte antigens is associated with outcomes in haploidentical stem cell transplantation. Blood Adv. 2020:4(15):3474-3485.

23. Kramer CSM, Israeli M, Mulder A, et al. The long and winding road towards epitope matching in clinical transplantation. Transpl Int. 2019;32(1):16-24.

24. Rimando J, Slade M, DiPersio JF, et al. HLA epitope mismatch in haploidentical transplantation is associated with decreased relapse and delayed engraftment. Blood Adv. 2018;2(24):3590-3601.
25. Geneugelijk K, Thus KA, Spierings E Predicting alloreactivity in transplantation. J Immunol Res. 2014;2014:159479.

26. Oran B, Saliba RM, Carmazzi Y, et al. Effect of nonpermissive HLA-DPB1 mismatches after unrelated allogeneic transplantation with in vivo T-cell depletion. Blood. 2018;131(11):1248-1257.

27. Duquesnoy RJ. Reflections on HLA epitopebased matching for transplantation. Front Immunol. 2016;7:469.

28. Crivello P, Zito L, Sizzano F, et al. The impact of amino acid variability on alloreactivity defines a functional distance predictive of permissive HLA-DPB1 mismatches in hematopoietic stem cell transplantation. Biol Blood Marrow Transplant. 2015;21(2):233241.

29. Vickers AJ, Elkin EB. Decision curve analysis: a novel method for evaluating prediction models. Med Decis Making. 2006;26(6):565574.

30. Vickers AJ, van Calster B, Steyerberg EW. A simple, step-by-step guide to interpreting decision curve analysis. Diagn Progn Res. 2019;3:18.

31. Rimando J, Slade M, DiPersio JF, et al. The Predicted Indirectly Recognizable HLA Epitopes (PIRCHE) score for HLA class I graft-versus-host disparity is associated with increased acute graft-versus-host disease in haploidentical transplantation with posttransplantation cyclophosphamide. Biol Blood Marrow Transplant. 2020;26(1):123131.

32. Fleischhauer K, Ahn KW, Wang HL, et al Directionality of non-permissive HLA-DPB1 T-cell epitope group mismatches does not improve clinical risk stratification in $8 / 8$ matched unrelated donor hematopoietic cell transplantation. Bone Marrow Ttransplant. 2017;52(9):1280-1287.

33. Gagne K, Loiseau P, Dubois V, et al. Is there any impact of HLA-DPB1 disparity in 10/10 HLA-matched unrelated hematopoietic SCT? Results of a French multicentric retrospective study. Bone Marrow Ttransplant. 2015;50(2):232-236

34. Petersdorf EW, Bengtsson M, De Santis D, et al. Role of HLA-DP expression in graft-versus-host disease after unrelated donor transplantation. J Clin Oncol. 2020;38(24):27122718.

35. Arai Y, Jo T, Matsui H, Kondo T, TakaoriKondo A. Efficacy of antithymocyte globulin for allogeneic hematopoietic cell transplantation: a systematic review and metaanalysis. Leuk Lymphoma. 2017;58(8):18401848.

36. Kanakry CG, O'Donnell PV, Furlong T, et al. Multi-institutional study of post-transplantation cyclophosphamide as single-agent graft-versus-host disease prophylaxis after 
allogeneic bone marrow transplantation using myeloablative busulfan and fludarabine conditioning. J Clin Oncol. 2014;32(31): 3497-3505.

37. Ruggeri A, Sun Y, Labopin M, et al. Posttransplant cyclophosphamide versus antithymocyte globulin as graft- versus-host disease prophylaxis in haploidentical transplant. Haematologica. 2017;102(2):401-410.

38. Geneugelijk K, Thus KA, van Deutekom HWM, et al. Exploratory study of predicted indirectly recognizable HLA epitopes in mismatched hematopoietic cell transplantations. Front Immunol. 2019;10:880.

39. van Seventer GA, Huis B, Melief CJ, Ivanyi P. Fine specificity of human HLA-B7-specific cytotoxic T-lymphocyte clones. I. Identification of HLA-B7 subtypes and his- totopes of the HLA-B7 cross-reacting group Hum Immunol. 1986;16(4):375-389.

40. Smith KD, Epperson DF, Lutz CT. Alloreactive cytotoxic T-lymphocytedefined HLA-B7 subtypes differ in peptide antigen presentation. Immunogenetics. 1996;43(1-2):27-37.

41. Hiraiwa M, Yamamoto J, Matsumoto K, et al. T cell can recognize the allospecificities formed by the substitution of amino acids associated with HLA-Bw4/Bw6 public epitopes. Hum Immunol. 1991;32(1):41-45.

42. Otten HG, Calis JJ, Kesmir C, van Zuilen $\mathrm{AD}$, Spierings E. Predicted indirectly recognizable HLA epitopes presented by HLADR correlate with the de novo development of donor-specific HLA IgG antibodies after kidney transplantation. Hum Immunol.
2013;74(3):290-296

43. Thus KA, de Hoop TA, de Weger RA, Bierings $\mathrm{MB}$, Boelens JJ, Spierings $\mathrm{E}$ Predicted indirectly recognizable HLA epitopes class I promote antileukemia responses after cord blood transplantation: indications for a potential novel donor selection tool. Biol Blood Marrow Transplant. 2016;22(1):170-173.

44. Stenger W, Kunkele A, Niemann M, et al. Donor selection in a pediatric stem cell transplantation cohort using PIRCHE and HLA-DPB1 typing. Pediatr Blood Cancer. 2020;67(3):e28127.

45. Thus KA, Te Boome L, Kuball J, Spierings E. Indirectly recognized HLA-C mismatches and their potential role in transplant outcome. Front Immunol. 2014;5:210. 